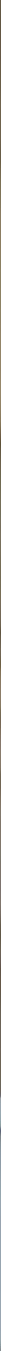

\title{
Do ADHD and ASD symptoms have similar characteristics in childhood and young adulthood?
}

\author{
By Dr. Jessica Edwards
}

Attention-deficit/hyperactivity disorder (ADHD) and autism spectrum disorder (ASD) are generally considered early-onset disorders. As a result, most research has focused on children, although it is now widely recognised that adults are also affected. ${ }^{1}$ Previous studies comparing ADHD and ASD symptoms in adulthood to childhood have observed some differences, such as less of a male excess ${ }^{2}$. However, this finding could reflect methodological issues including changing the informant from parent to self, different 'child' and 'adult' measures, delayed detection in females, or referral bias in adult clinic-based studies.

Now, Lucy Riglin and colleagues in the UK have investigated whether ADHD and ASD traits in young adulthood show similar characteristics to those reported in childhood. To do so, they harnessed data from a longitudinal study (ALSPAC) that followed the same individuals during childhood into young adulthood and used many of the same measures across this timeframe. They examined associations with other neurodevelopmental problems (IQ, reading and spelling ability, pragmatic language and communication) and examined the pattern of associations with neuropsychiatric polygenic risk scores (focussing on disorders where previous research suggests genetic overlap in childhood: ADHD, ASD, schizophrenia, depression and anxiety). The researchers also explored whether the patterns of association differed when using self-reports compared to parent-reports. 
